\title{
NCCN Virtual Patient Advocacy Summit: Cancer Across the Lifespan
}

Kara Martin, $\mathrm{MPH}^{1}$; Alyssa A. Schatz, MSW${ }^{1}$; Jan S. White, $\mathrm{BA}^{2}$; Hyman Muss, MD${ }^{3}$; Aarati Didwania, MD, MSCl${ }^{4}$; Leigh Gallo, JD'; and Robert W. Carlson, $\mathrm{MD}^{1}$

\begin{abstract}
Patients with cancer have widely divergent experiences throughout their care from screening through survivorship. Differences in care delivery and outcomes may be due to varying patient preferences, patient needs according to stage of life, access to care, and implicit or explicit bias in care according to patient age. NCCN convened a series of stakeholder meetings with patients, caregivers, and patient advocacy groups to discuss the complex challenges and robust opportunities in this space. These meetings informed the NCCN Virtual Patient Advocacy Summit: Cancer Across the Lifespan held on December 10, 2020, which featured a keynote presentation, multidisciplinary panels, and presentations from patient advocacy organizations. This article encapsulates and expounds upon the findings from the stakeholder meetings and discussions during the summit.
\end{abstract}

J Natl Compr Canc Netw 2021;19(12):1395-1400 doi: 10.6004/jnccn.2021.7087

\footnotetext{
${ }^{1}$ National Comprehensive Cancer Network, Plymouth Meeting, Pennsylvania; ${ }^{2}$ Patient Advocate, Jan Says Onward, Newark, Delaware; ${ }^{3}$ University of North Carolina Lineberger Comprehensive Cancer Center, University of North Carolina School of Medicine, Chapel Hill, North Carolina; and ${ }^{4}$ Robert H. Lurie Comprehensive Cancer Center of Northwestern University, Northwestern University Feinberg School of Medicine, Chicago, Illinois.
}

Patients with cancer have widely divergent experiences throughout their care from screening through survivorship. Differences in care delivery and outcomes may be due to varying patient preferences, patient needs according to stage of life, access to care, and implicit or explicit bias in care according to patient age. To explore the complex challenges and robust opportunities in this space, NCCN hosted the NCCN Virtual Patient Advocacy Summit: Cancer Across the Lifespan. The summit featured a keynote presentation, multidisciplinary panels, and presentations from patient advocacy organizations (participants are listed in supplemental eAppendices 1 and 2, available with this article at JNCCN.org). Panelists provided insights and recommendations; however, the goal of this program was to highlight key issues and not to obtain consensus.

In an effort to keep the patient voice at the center of the event, NCCN convened a series of stakeholder meetings with patients, caregivers, and patient advocacy groups to discuss the ways in which patient experience varies across different stages of life and across the care continuum. Participants highlighted overarching barriers to cancer care in the United States, including inadequate health coverage, delayed testing and screening, inconsistent guideline adherence, and more. However, they also provided stark analyses of the unique barriers faced by individual demographic groups, and the way those barriers are compounded for patients who fall into one or more groups. The general sentiment among patient advocates is that in order to alleviate barriers, whether they are shared or unique, changes must be made within the healthcare system to promote better attention to patient-specific needs and preferences.

\section{Living and Thriving Through Cancer}

No one person is the same. Each individual has varying goals, obstacles, and levels of social support to navigate life's ups and downs. This was made clear by the keynote speaker Jan S. White, a survivor of stage IV non-Hodgkin lymphoma, as she described her experience navigating

See JNCCN.org for supplemental online content. 
cancer treatment in a healthcare system that assumes or generalizes much too often. In one of many examples of assumptions experienced through her cancer "adventure," Ms. White described the way her providers asked about her level of physical activity prediagnosis and then were stunned at her response. Prediagnosis, she was teaching kickboxing, studying for her 4th degree karate black belt, and running a successful small business. This conversation may not have been so frustrating for Ms. White if it did not directly connect to her care team's assumptions about who she was, what she wanted, and what she could achieve. To optimize outcomes, healthcare teams must have comprehensive, ongoing discussions with patients about what their treatment options are while adhering to professional guidelines, being mindful of patient preferences, and acknowledging their own potential for bias.

Some patients report not getting enough information when they are diagnosed, and do not feel involved in their care planning. Others report wanting a stronger focus on the emotional side effects of their treatment whereas their provider only addresses the physical manifestations of their cancer. In each case, the opportunity for the patient to express what they need is missing. Shared decision-making is especially useful in cancer care and can open up the lines of communication between patients and their care team. ${ }^{1,2}$

Shared decision-making may also decrease implicit bias in cancer care. Age, race, and gender bias in cancer care can negatively impact a patients' outcomes, experiences, and general well-being, and require significant investigation and intervention..$^{3-5}$ As Ms. White put it, "Ask your patients what their goals are for how they want to live posttreatment. Frame the conversation around those goals, instead of limitations. Not everyone fits into nice, neat, expected boxes; many patients will surprise you."

\section{Addressing Age-Specific Challenges in Cancer Care}

\section{Children, Teenagers, and Young Adults}

Pediatric and young adult patients face unique challenges including disparities in advances in pediatric cancer research, late-term effects of treatment, and unique survivorship care planning needs. A significant barrier to advances in care for pediatric and young adult patients is a dearth of therapies tested and approved specifically for children and young adults. ${ }^{6}$ Due to the lack of protocols for many rare pediatric cancers, the default is to follow the adult protocol for the rare disease. In 2019, NCCN began publishing several guidelines for pediatric care, which are developed to assist patients, caregivers, and healthcare professionals in the decision-making process. ${ }^{7}$
Patients diagnosed with cancer as young adults face daunting questions when determining treatment plans: Can I go away to college? Will I be able to have kids? Can/Should I get married? How will I transition back to life/school/work? These questions stem from systemic barriers including gaps in insurance coverage for fertility preservation and scarce resources and support for young adults transitioning to work or college while managing a cancer diagnosis. For young adults who are already a part of the workforce, the threat of losing their income during treatment may be significant and may lead them to delay or refuse treatment entirely. Dr. Aarati Didwania, Professor of Medicine and Medical Education and Director of the Survivors Taking Action and Responsibility (STAR) Program, Robert H. Lurie Comprehensive Cancer Center of Northwestern University, reflected that there is often a compression of time that does not allow for as much conversation a patient may need to process treatment options and identify priorities.

Access to and management of relevant medical information from active treatment to survivorship care is often challenging for children and teenagers, because treatment plans are typically communicated to patients' parents or guardians. Children and teenagers also may be most at risk for unwanted late effects, because late effects are not typically at the forefront of the mind when caring for young patients. Two pieces of legislation passed in 2017, the Research to Accelerate Cures and Equity for Children Act (RACE Act) and the Survivorship, Treatment, Access, and Research Act (STAR Act), amend previous laws to prioritize the advancement of pediatric cancer research and improve care coordination throughout the lifespan. ${ }^{6,8}$ In a fireside chat, US Representative Buddy Carter (R-GA) and US Senator Chris Van Hollen (D-MD) shared that these bills stemmed from dedicated grassroots efforts led by affected families and individuals, and were passed in a bipartisan manner. The congressmen outlined a few strategies for building upon the passage of the RACE and STAR Acts, including a need to record best practices during implementation, measure impact, and continue addressing pediatric cancer issues within congressional caucuses and committees on cancer. Senator Van Hollen and Representative Carter encouraged oncology stakeholders to continue influencing legislation by sharing their lived experiences with cancer and applauded stakeholders for their courage and commitment to making their voice heard.

\section{Adults and Older Adults}

Adulthood spans several decades and looks different for each individual depending on their occupation, family status, education, and zip code. Examples of unique challenges for adult patients are fertility issues and delayed diagnosis, and challenges for older adult patients may 
include frequent comorbidities, underrepresentation in clinical trials, ageism, and situational barriers to care.

Many cancer treatments may have significant implications for an individual's fertility following treatment completion. Given these implications, clinicians, patient advocacy organizations, and other stakeholders must ensure that patients are informed of potential toxicities and late-term effects of treatment and are aware of their options for fertility preservation coverage. As of early 2021, 28 states have enacted or introduced legislation that requires payer coverage of fertility preservation treatment for enrollees undergoing cancer treatment, but 9 of those states' legislation is now inactive. ${ }^{9}$

Adults also face barriers to timely and accurate diagnosis for certain diseases, such as ovarian, prostate, or colorectal cancer (CRC). The CDC estimates that $68 \%$ of deaths from CRC could be avoided if all eligible people got screened. ${ }^{10}$ Patients aged $<50$ years are 58\% more likely than older patients to be diagnosed with stage III or IV CRC. ${ }^{11}$ Studies suggest that this is caused by insufficient access to healthcare and a lack of awareness among patients and providers about the signs and symptoms of the disease. ${ }^{11}$ Healthcare providers must be cognizant of key symptoms of CRC in younger populations to screen, diagnose, and treat it.

Older adult patients with cancer require a thoughtful approach to treatment and care management due to the population's unique needs, increased likelihood of comorbid conditions such as heart disease and diabetes, and increased likelihood of experiencing certain toxicities. As of 2018, more than half of new cancer diagnoses were for patients aged $>65$ years. ${ }^{12}$ However, this population is significantly underrepresented in clinical trials, which play an important role in ensuring drug efficacy and safety and fostering innovation. ${ }^{12}$ Recognizing this challenge, in March 2020, the FDA released Draft Guidance for Industry titled "Inclusion of Older Adults in Cancer Clinical Trials." 13 This guidance outlines solutions the FDA may be open to including flexible approaches to trial design, such as open label safety studies or trials stratified by age. With a new administration as of January 2021, further stakeholder engagement will be critical to expanding clinical trial access and advancing patientcentered solutions.

Older adults also face several barriers to care due to ageism and implicit bias. ${ }^{14}$ For example, providers sometimes set recovery goals lower for an older adult than they would for another patient simply based on age, as opposed to asking the patient what their recovery goals are directly. Danielle S. Pardue, a dedicated caregiver and patient advocate, witnessed this firsthand throughout her mother's cancer treatment. "My mom - now in her mid70 s-is someone you can count on to be the first person on the dance floor at a wedding. And yet, in lieu of her cancer diagnosis and treatment, her physical therapy team told her she would never walk again. Such assumptions can be made among the most well-intentioned, kind, and professional providers." Providers Hyman Muss, MD, from the University of North Carolina Lineberger Comprehensive Cancer Center, and Efrat Dotan, MD, from Fox Chase Cancer Center, Chair of the NCCN Older Adult Oncology Panel, shared Ms. Pardue's sentiment that discussions about quality of life versus quantity of life are just as important to have with older adults as they are with young people.

Lastly, older adults may face one or more of the following barriers to care: discomfort with technology, restricted mobility, low socioeconomic status, and isolation from family and friends. ${ }^{15-17}$ The older patient population also has a propensity toward white coat syndrome, potentially leading them to make treatment decisions without having full information. For these older adults, the idea of being assertive with someone in a position of authority can be intimidating. The assistance of a caregiver can alleviate many of these challenges; however, support for caregivers in the United States is lacking.

\section{Addressing Inequities in Cancer Care}

Inequities in cancer care and population-specific challenges are important to consider as stakeholders work to provide quality, effective, efficient, and accessible cancer care across the lifespan. Two specific challenges highlighted at the Patient Advocacy Summit were race/ethnicity and language.

Historic and present-day inequities have led many people of color (POC), specifically those who are Black, to mistrust the health system. Historically, the health system has exploited Black patients for research advancements and used their patient information without consent. ${ }^{18-21}$ Present day racial and ethnic minorities still disproportionately experience unconscious bias, restricted access to care, and poor health outcomes in the United States. ${ }^{22-25}$ Because racism is pervasive throughout all US institutions, POC also face injustices in housing, education, and workplaces; each of these factors are compounded, leaving POC at a significant disadvantage compared with their White counterparts. ${ }^{26,27}$ Although morbidity and mortality gaps have been shrinking in recent years, Black individuals still have the highest death rate and the lowest survival rate of any racial or ethnic group for most cancers. ${ }^{28}$

Non-English-speaking patients and those who speak English as a Second Language (ESL) also experience enhanced access barriers, because the healthcare system does not currently provide adequate resources or strategies to support them..$^{29} \mathrm{~A}$ dearth of appropriate resources to meet the needs of this patient population may erode the patient-provider relationship, increase the likelihood of health miscommunication, and infringe on patient 
privacy. ${ }^{30}$ Darcie Green, CEO/Executive Director at Latinas Contra Cancer, shared that ESL patients often resort to using a family member to help with translationsometimes because that is preferred and sometimes because there is no other available option. This burdens the patient and their family members and causes health privacy issues that must be addressed to improve patient outcomes and experiences in oncology.

\section{Solutions to Improve Care Across the Patient Lifespan}

\section{Patient Advocacy Solutions}

A digital booklet of patient advocacy resources was developed in conjunction with this summit to highlight the range of resources available to patients with cancer and their loved ones. The booklet included information from 25 patient advocacy organizations on their age-specific offerings. ${ }^{31}$ Patient advocacy resources were also featured during the summit, outlined as follows.

- Sarah Miretti Cassidy presented on the Cancer Hope Network's Peer Support Program ${ }^{32}$ available to adult cancer patients and their loved ones. Clients of this free and confidential program are matched with a Cancer Hope Network Support Volunteer and referred to a wide array of community resources based on their unique needs.

- Dottie Shead, MS, discussed age-related NCCN Guidelines for Patients, ${ }^{33}$ including those for pediatric acute lymphoblastic leukemia (ALL), adolescents and young adults with cancer, and survivorship care. NCCN Guidelines for Patients are developed to empower people to make informed decisions at each step of their cancer journey and can be accessed online at NCCN.org, on the NCCN Patient Guides for Cancer app, and in print via Amazon.com.

- Molly McDonnell reviewed Fight Colorectal Cancer's Catalyst State-by-State Advocacy Program, ${ }^{34}$ which aims to increase access and reduce barriers to CRC screening by supporting state coalitions with grant funding and technical assistance. As of December 2020, the Catalyst program helped advance CRC screening efforts in California, Louisiana, Arkansas, and Colorado, with goals to expand to Texas, Nebraska, Rhode Island, and Kentucky in 2021.

- Andrea Conners, MS, showcased the Patient Empowerment Network's digital sherpa Program, ${ }^{35}$ which consists of in-person and virtual workshops to help older adults use technology to their advantage as they navigate their cancer journey. Main course objectives of the digital sherpa Program include how to access the internet, identify credible resources and websites, and access patient portals.

Patient advocacy organizations are leading the way to develop patient-centered solutions. However, systemic policy and practice changes are still needed to address the myriad challenges that patience face throughout the cancer care continuum.

\section{Policy and Practice Solutions}

Throughout the summit, panelists discussed dynamic methods to reduce barriers and improve patient health across the lifespan, such as addressing research and development gaps, addressing implicit and explicit bias, strengthening health education and communication, expanding and diversifying healthcare teams, and improving health care planning and coordination. These policy and practice solutions were explored in a multidisciplinary stakeholder roundtable to close out the event.

Expanding clinical trial participation across race and age is critical to addressing research and development gaps. According to Dr. Maura Dickler, Eli Lilly and Company, testing drugs and therapies in diverse populations is necessary to glean the different ways people metabolize medications. The Clinical Treatment Act, H.R. 913, a bill requiring state Medicaid programs to cover routine costs of care during clinical trials, was highlighted by 2 summit panelists as a way to increase participation in trials. ${ }^{36}$ Phylicia L. Woods from Cancer Support Community and Alyssa Schatz from NCCN explained that all other major payers cover these costs, and that Medicaid beneficiaries are unlikely to participate in clinical trials without similar support. H.R. 913 was ultimately included in the Consolidated Appropriations Act, which passed and became law on December 27, 2020, with an effective date of January $1,2022 .^{37}$

Addressing implicit and explicit bias at the individual, community, and systems levels would greatly improve patient health across the lifespan. Health professionals must be thoroughly educated about bias in healthcare and its impact on patients. At the systems level, this could be accomplished by incorporating antiracist and antibias education into medical student and resident curricula. Additionally, supplementary antibias education could be offered as continuing education for providers who graduated before such education standards were developed.

Strengthening health education and communication is pivotal to addressing patient needs and preferences across the lifespan. This would involve improving patient-provider communication, including patients and their caretakers in shared decision-making, and providing translation services for those who need them. Using existing tools such as the Geriatric Assessment from the Cancer and Aging Research Group and the various assessments endorsed by the International Society of 
Geriatric Oncology can help achieve a baseline understanding of individual patient needs. ${ }^{38,39}$

The oncologist should be one of many healthcare professionals on a patient's healthcare team. Cancer treatment and survivorship care are multifaceted, and a high volume of patient support is necessary. Assigning value to social workers, nurses, patient navigators, financial advisors, and caregivers as a part of the team can enhance patient care. Ms. Pardue suggested that in order for caregivers to provide sufficient support to their loved ones, they must be provided with financial, educational, and emotional resources. A caregiver can be any age and likely faces unique challenges based on their current stage of life; their decision to become a caregiver will depend on their comfort level with the healthcare system, relationship to the patient, financial security, and general availability.

Improving healthcare planning and coordination would be especially impactful for patients and survivors of cancer. The Cancer Care Planning and Communications Act (CCPCA), H.R. 3835, introduced in July 2019, incentivized the development of personalized cancer care plans for Medicare beneficiaries. ${ }^{40}$ If CCPCA is reintroduced and passed by the 117th Congress, more funds will become available to reimburse Medicare providers for supplying patients with a written plan for managing their diagnosis, treatment, and survivorship. Policymakers and other stakeholders could make an even larger impact on care planning and coordination in the United States by expanding patient access to electronic health records (EHRs) and improving EHR interoperability for smoother patient transitions from oncology to primary care.

\section{Conclusions}

Each patient with cancer has varying goals, obstacles, and levels of social support throughout their cancer "adventure." Several barriers exist for the healthcare system to adequately and consistently meet patient needs. Patient advocates and other stakeholders identified concrete ways to address these barriers at the individual, practice, and policy levels. The surplus of opportunities to improve cancer across the lifespan highlights the immense potential for positive change if the cancer community continues to innovate and collaborate. However, without patient input, this innovation and collaboration will yield minimal results. Science, practice, and policy experts must partner with patients to make comprehensive policy and practice solutions that address complex patient needs.

Submitted May 26, 2021; final revision received August 11, 2021; accepted for publication September 8, 2021

Disclosures: The authors have disclosed that they have no financial interests, arrangements, or affiliations with the manufacturers of any products discussed in this article or their competitors.

Correspondence: Alyssa A. Schatz, MSW, National Comprehensive Cancer Network, 3025 Chemical Road, Suite 100, Plymouth Meeting, PA 19426. Email: schatz@nccn.org

\section{References}

1. Bomhof-Roordink H, Fischer MJ, van Duijn-Bakker N, et al. Shared decision making in oncology: a model based on patients', health care professionals', and researchers' views. Psychooncology 2019;28:139-146.

2. Faiman B. Shared decision-making in cancer treatment 'worth the effort'. Accessed September 1, 2021. Available at: https://www.healio.com/ news/hematology-oncology/20180313/shared-decisionmaking-in-cancertreatment-worth-the-effort

3. Joszt L. Implicit biases have an explicit impact on healthcare outcomes. Accessed September 1, 2021. Available at: https://www.ajmc.com/view/ implicit-biases-have-an-explicit-impact-on-healthcare-outcomes

4. Efforts to reduce racial disparities in cancer care aim to 'make the invisible visible'. Accessed September 1, 2021. Available at: https://www.healio.com/news/hematology-oncology/20190514/efforts-to-reduce-racialdisparities-in-cancer-care-aim-to-make-the-invisible-visible

5. Ben Zaniello M. Implicit bias and racial disparities of care: recognizing and addressing the role of implicit bias in vulnerable patient care. Becker's Hospital Review. Accessed September 1, 2021. Available at: https://www.beckershospitalreview.com/care-coordination/implicit-biasand-racial-disparities-of-care-recognizing-and-addressing-the-role-ofimplicit-bias-in-vulnerable-patient-care.html

6. Alliance for Childhood Cancer. Alliance for Childhood Cancer Celebrates Reintroduction of the Childhood Cancer STAR Act in the 115th Congress. Accessed September 1, 2021. Available at: https://www. allianceforchildhoodcancer.org/legislative-activity/newsroom.html/article/ 2017/02/03/alliance-for-childhood-cancer-celebrates-reintroduction-ofthe-childhood-cancer-star-act-in-the-115th-congress

7. National Comprehensive Cancer Network. NCCN Guidelines. Treatment by cancer type. Accessed September 1, 2021. Available at: https:// www.nccn.org/guidelines/category_1
8. Kids V Cancer. RACE for Children Act becomes law! Accessed September 1, 2021. Available at: www.kidsvcancer.org/race-for-children-act/

9. Alliance for Fertility Preservation. State laws \& legislation. Accessed September 1, 2021. Available at: https://www. allianceforfertilitypreservation.org/advocacy/state-legislation

10. Sharma KP, Grosse SD, Maciosek MV, et al. Preventing breast, cervical, and colorectal cancer deaths: assessing the impact of increased screening. Prev Chronic Dis 2020;17:E123.

11. Facts and stats. Accessed September 1, 2021. Available at: https:// fightcolorectalcancer.org/about-colorectzal-cancer/general-information/ facts-stats/. Fight Colorectal Cancer.

12. Sharpless NE. The challenging landscape of cancer and aging: charting a way forward. National Cancer Institute. Accessed September 1, 2021 Available at: https://www.cancer.gov/news-events/cancer-currents-blog/ 2018/sharpless-aging-cancer-research

13. Inclusion of Older Adults in Cancer Clinical Trials; Draft Guidance for Industry. Fed Regist 2020;85:13167-13168.

14. Wyman MF, Shiovitz-Ezra S, Bengel J. Ageism in the health care system providers, patients, and systems. In: Ayalon L, Tesch-Romer C, eds. Contemporary Perspectives on Ageism. International Perspectives on Aging, volume 19. Cham, Switzerland: Springer; 2018:193-212.

15. Smith A. Older adults and technology use. Pew Research Center. Accessed September 1, 2021. Available at: https://www.pewresearch.org/internet/2014/04/03/older-adults-and-technology-use/

16. McMaughan DJ, Oloruntoba O, Smith ML. Socioeconomic status and access to healthcare: interrelated drivers for healthy aging. Front Public Health 2020;8:231

17. Centers for Disease Control and Prevention. Loneliness and social isolation linked to serious health conditions. Accessed September 1, 2021. 
Available at: https://www.cdc.gov/aging/publications/features/lonelyolder-adults.html

18. Stein R. All Things Considered. Troubling history in medical research still fresh for Black Americans. October 25, 2017. Accessed September 1, 2021. Available at: https://www.npr.org/sections/health-shots/2017/10/ 25/556673640/scientists-work-to-overcome-legacy-of-tuskegee-studyhenrietta-lacks

19. Ko L. Unwanted sterilization and eugenics programs in the United States. Accessed September 1, 2021. Available at: https://www.pbs.org/independentlens/blog/unwanted-sterilization-and-eugenics-programs-in-theunited-states/

20. Ojanuga D. The medical ethics of the 'father of gynaecology', Dr J Marion Sims. J Med Ethics 1993;19:28-31.

21. Centers for Disease Control and Prevention. The U.S. Public Health Service Syphilis Study at Tuskegee. Accessed September 1, 2021. Available at: https://www.cdc.gov/tuskegee/index.html

22. National Academies of Sciences, Engineering, and Medicine. Communities in action: pathways to health equity. Washington, DC: National Academies Press; 2017

23. National Research Council (US) Panel on Race, Ethnicity, and Health in Later Life; Bulatao RA, Anderson NB, eds. Understanding Racial and Ethnic Differences in Health in Late Life: A Research Agenda. Washington, DC: National Academies Press; 2004.

24. Artiga S, Orgera K. Key facts on health and health care by race and ethnicity. Accessed September 1, 2021. Available at: https://www.kff.org/ racial-equity-and-health-policy/report/key-facts-on-health-and-healthcare-by-race-and-ethnicity/

25. Carratala S, Maxwell C. Health disparities by race and ethnicity. Center for American Progress website. Accessed September 1, 2021. Available at: https://www.americanprogress.org/issues/race/reports/2020/05/07/ 484742/health-disparities-race-ethnicity/

26. Morgan JD, De Marco AC, LaForett DR, et al. What racism looks like: an infographic. Frank Poerter Graham Child Development Institute, University of North Carolina at Chapel Hill. Accessed September 1, 2021 Available at: https://fpg.unc.edu/sites/fpg.unc.edu/files/resources/otherresources/What\%20Racism\%20Looks\%20Like.pdf

27. Bailey ZD, Feldman JM, Bassett MT. How structural racism works-racist policies as a root cause of US racial health inequities. N Engl J Med 2021;384:768-773.

28. DeSantis CE, Miller KD, Goding Sauer A, et al. Cancer statistics for African Americans, 2019. CA Cancer J Clin 2019;69:211-233.
29. Cohen R. Required translators missing from many U.S. hospitals. Accessed September 1, 2021. Available at: https://www.reuters.com/article/us-health-translators/required-translators-missing-from-many-u-s-hospitals-idUSKCN10M29M

30. Allen MP, Johnson RE, McClave EZ, Alvarado-Little W. Language, interpretation, and translation: a clarification and reference checklist in service of health literacy and cultural respect. Accessed September 1, 2021. Available at: https://nam.edu/language-interpretation-and-translation-a-clarification-andreference-checklist-in-service-of-health-literacy-and-cultural-respect/

31. National Comprehensive Cancer Network. NCCN Digital Booklet of Patient Advocacy Resources. Virtual Patient Advocacy Summit: Cancer Across the Lifespan. Accessed September 1, 2021. Available at: https:// www.nccn.org/docs/default-source/oncology-policy-program/ cancer_across_life_span.pdf?sfvrsn=1b738f08_2

32. Cancer Hope Network. Frequently asked questions. Accessed September 1, 2021. Available at: https://www.cancerhopenetwork.org/who-we-are/ faq.html

33. National Comprehensive Cancer Network. Guidelines for Patients. Accessed September 1, 2021. Available at: https://www.nccn.org/ patients/guidelines/cancers.aspx

34. Catalyst State-by-State Advocacy Program. Accessed September 1, 2021 Available at: https://fightcolorectalcancer.org/advocacy/catalyst-program/

35. Patient Empowerment Network. Digitally Empowered and Digital Sherpa. Accessed September 1, 2021. Available at: https://powerfulpatients.org/ digitally-empowered-and-digital-sherpa/\#digitalsherpa

36. Clinical Treatment Act, HR 913, 116th Congress (2019-2020).

37. Consolidated Appropriations Act, 2021, HR 133, 116th Congress (2019-2020).

38. Cancer and Aging Research Group. Tools. Accessed September, 1, 2021. Available at: https://www.mycarg.org/?page_id=166

39. International Society of Geriatric Oncology. Comprehensive Geriatric Assessment (CGA) of the older patient with cancer. Accessed September 1, 2021. Available at: http://www.siog.org/content/comprehensive-geriatric-assessment-cga-older-patient-cancer

40. National Coalition for Cancer Survivorship. Cancer Care Planning and Communications Act (CCPCA). Accessed September 1, 2021. Available at: https://canceradvocacy.org/policy/cancer-care-planning-and-communications-act-ccpca/ 

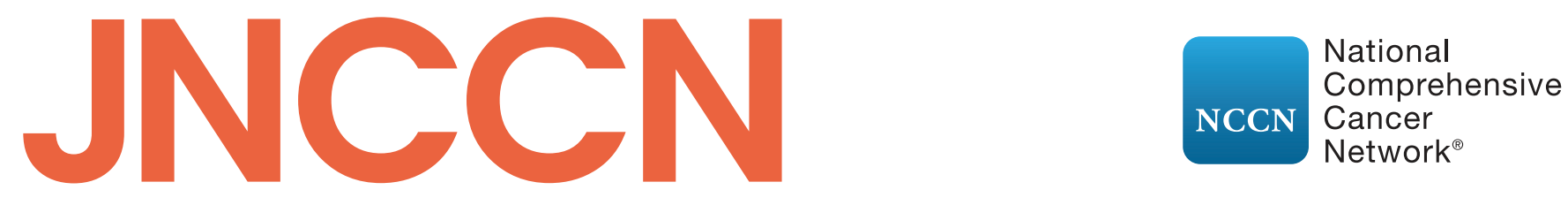

JOURNAL OF THE NATIONAL COMPREHENSIVE CANCER NETWORK

Supplemental online content for:

\section{NCCN Virtual Patient Advocacy Summit: Cancer Across the Lifespan}

Kara Martin, MPH; Alyssa A. Schatz, MSW; Jan S. White, BA; Hyman Muss, MD;

Aarati Didwania, MD, MSCl; Leigh Gallo, JD; and Robert W. Carlson, MD

J Natl Compr Canc Netw 2021;19(12):1395-1400

eAppendix 1: Stakeholder Meeting Participants

eAppendix 2: Summit Participants 


\section{eAppendix 1. Stakeholder Meeting Participants}

American Cancer Society Cancer Action Network

Cancer Hope Network

Cancer Support Community

Caregiver, North Carolina/New York

FORCE: Facing Our Risk of Cancer Empowered

Head and Neck Cancer Fund

HPV Alliance

Kidney Cancer Association

Latinas Contra Cancer

National Coalition for Cancer Survivorship

National Patient Advocate Foundation

NormaLeah Cancer Initiative

Patient Empowerment Network

Patient, Delaware

Patient, Pennsylvania

Research Advocacy Network

The Leukemia \& Lymphoma Society

Veterans Prostate Cancer Awareness

\section{eAppendix 2. Summit Participants}

Aarati Didwania, MD, MSCI, Robert H. Lurie Comprehensive Cancer Center of Northwestern University

Alyssa Schatz, MSW, National Comprehensive Cancer Network

Andrea Conners, MS, Patient Empowerment Network

Clifford Goodman, PhD, The Lewin Group

U.S. Representative Buddy Carter (R-GA)

Danielle S. Pardue, JD, Caregiver and Patient Advocate

Darcie Green, Latinas Contra Cancer

Dottie Shead, MS, National Comprehensive Cancer Network

Efrat Dotan, MD, Fox Chase Cancer Center

Gary J. Weyhmuller, MBA, SPHR, National Comprehensive Cancer Network

Hyman Muss, MD, University of North Carolina Lineberger Comprehensive Cancer Center

Jan S. White, BA, Cancer Survivor and Patient Advocate

Maura Dickler, MD, Eli Lilly and Company

Molly McDonnell, Fight Colorectal Cancer

Phylicia L. Woods, JD, MSW, Cancer Support Community

Robert W. Carlson, MD, National Comprehensive Cancer Network

Sarah Miretti Cassidy, Cancer Hope Network

U.S. Senator Chris Van Hollen (D-MD) 